\title{
Fellowship training, workload, fatigue and physical stress: a prospective observational study
}

\author{
Christopher S. Parshuram, Sonny Dhanani, Joel A. Kirsh, Peter N. Cox
}

13 See related article page 975

\section{Abstract}

Background: Fatigue in physician trainees may compromise patient safety and the well-being of the trainees and limit the educational opportunities provided by training programs. Anecdotal evidence suggests that the on-call workload and physical demands experienced by trainees are significant despite dutyhour regulation and support from nursing staff, other trainees and staff physicians.

Methods: We measured the workload and the level of fatigue and physical stress of 11 senior fellows during 35 shifts in the critical care unit at the Hospital for Sick Children in Toronto. We determined number of rostered hours, number of admissions and discharges, number and type of procedures, nurse:patient ratios and related measures of workload. Fellows self-reported the number of pages they received and the amount of time they slept. We estimated physical stress by using a commercially available pedometer to measure the distance walked, by using ambulatory electrocardiographic monitoring to determine arrhythmias and by determining urine specific gravity and ketone levels to estimate hydration.

Results: The number of rostered hours were within current Ontario guidelines. The mean shift duration was 25.5 hours (range 24-27 hours). The fellows worked on average 69 hours (range 55-106) per week. On average during a shift, the fellows received 41 pages, were on non-sleeping breaks for 1.2 hours, slept 1.9 hours and walked $6.3 \mathrm{~km}$. Ketonuria was found in participants in 7 (21\%) of the 33 shifts during which it was measured. Arrhythmia (1 atrial, 1 ventricular) or heart rate abnormalities occurred in all 6 participants. These fellows were the most senior in-house physician for a mean of 9.4 hours per shift and were responsible for performing invasive procedures in two-thirds of their shifts.

Interpretation: Established Canadian and proposed American guidelines expose trainees to significant on-call workload, physical stress and sleep deprivation.

CMAJ 2004;170(6):965-70 ecognition that clinician fatigue may promote ineffective care and lead to adverse patient outcomes ${ }^{1-3}$ has provided a compelling rationale for the enforcement of resident duty-hour guidelines. Although these guidelines permit trainees to work more hours - both continuously and cumulatively — than other nonphysician groups, ${ }^{2}$ they represent an effort to reduce physician work hours and promote patient safety.

The current Professional Association of Internes and Residents of Ontario-Ontario Council of Teaching Hospitals (PAIRO-OCOTH) agreement ${ }^{4}$ has been in place since July 2000. This agreement limits residents to a maximum of 7 in-house on-call periods of up to 24 hours (plus a handover period) in 28 days. Call periods are nonconsecutive, should not include more than 2 weekend days in the 28-day cycle and are followed by "relief of service until the next working day."

The PAIRO-OCOTH agreement also requires the provision of sleeping facilities - implicitly suggesting that oncall trainees may rest during in-house call. Rest may mitigate the effects of long duty hours. ${ }^{2}$ However, experience suggests that on-call shifts are physically demanding and that opportunities for rest and sleep are limited. ${ }^{5}$

Although recognition of the relation between trainee duty-hours and fatigue and patient and physician safety is increasing, ${ }^{2,5,6}$ direct assessment of workload, physical stress and the impact of staff and junior physician support has been limited. To evaluate the work environment of trainees, we conducted a prospective study in the critical care unit of a university-affiliated tertiary care centre.

\section{Methods}

The Hospital for Sick Children is a 300-bed university-affiliated teaching hospital. Its pediatric critical care unit (PCCU) is a 36bed multidisciplinary unit staffed by 150 nurses, 45 respiratory therapists, 12 fellows, 1 or 2 residents and 5 full-time staff physicians (intensivists). It is a closed unit, in that intensivists from the unit assume primary responsibility for admitted patients. Each year, 1700 children are admitted, for a total of about 6000 patientdays. The on-call intensivist is directly responsible for the supervision and support of trainees.

The on-call structure involves 1 or 2 staff intensivists, who are not required to stay in the hospital, and 1 senior fellow, a junior fellow and, occasionally, a third physician (the rotating resident), all of whom remain in the hospital. Between the morning rounds $(7: 30 \mathrm{am})$ and the sign-out rounds $(4: 30 \mathrm{pm})$ on weekdays, the oncall physician team is joined by the other 6-10 fellows and residents who are not post-call. On weekends, the incoming and out- 
going physician teams overlap during handover; otherwise, the members of the on-call team are the only physicians in the PCCU.

Senior fellows are usually international medical graduates (as there are insufficient Canadian critical care medicine trainees to fill the available positions) with 6-12 years of postgraduate training, including completion of formal training requirements in one or more of pediatric medicine, anesthesia, surgery and intensive care in their country of origin. They have completed a minimum of 1 year in pediatric intensive care medicine, and their clinical performance in the Hospital for Sick Children's PCCU has been assessed as appropriate for this designation. Admission to the hospital's pediatric critical care medicine fellowship program is highly competitive. The $8-10$ international trainees admitted to the program each year are often in the final stages of their clinical training and bring many skills and experiences to the program during their 6- or 12-month rotations. The senior fellow is the most senior in-house physician. He or she is responsible for coordination and support of the other on-call physicians, management of calls from outside the unit and communication with the intensivists.

Measures of patient-related physician workload, physiologic stress and unit staffing were recorded for up to 5 shifts for each fellow. In addition, two 4-week periods were chosen at random from the senior fellow's roster and the mean and total number of hours rostered per week calculated. Work practice was compared with the 2000 PAIRO-OCOTH guidelines. ${ }^{4}$

Data were collected using a standardized form, which was given to senior fellows at the start of their shift and reviewed for completeness at the end of the shift. Additional administrative and electrocardiographic data were added to the form before data entry and analysis.

To measure workload, we obtained patient numbers and acuity directly from the PCCU administrative database. The number of children receiving mechanical ventilation, extracorporeal membrane oxygenation, hemodialysis and high-frequency oscillation was recorded, and initiation of these therapies during the call period was noted. Senior fellows prospectively recorded the number and type of procedures they performed or supervised, the number of patients they transported outside the unit, the number of pages to them and the occurrence of cardiorespiratory arrest and death in patients they managed. At the end of each shift, participants evaluated how busy and how psychologically stressful the on-call period was using a 7 -point numerical scale ( $1=$ least, 7 = worst $)$ in comparison with the participants' previous experience.

To determine physiologic stress, we gave senior fellows a commercially available pedometer (Sportline Pedometer 360, New Balance, Boston) to measure the distance walked during each shift. Stride length was calibrated, and the number of steps taken and distance walked during the shift were recorded. In addition, participants recorded the duration of breaks and total sleeping time. Urinalysis for specific gravity and ketones was performed at the end of the shift (MultiStix 10 SG, Bayer, Toronto, Ont.). Ambulatory electrocardiographic (Holter) monitoring was performed where feasible using Marquette Series 8500 recorders (GE Marquette Medical Systems, Milwaukee).

To determine the impact of staff support, the number of nursing staff and nurse:patient ratios were calculated. The number of in-house physicians and intensivists on-call, the time of their departure, subsequent communication between senior fellows and intensivists and the return of intensivists to the unit after departure were also documented.

Descriptive statistics were used to represent the data. Compar- isons between shifts with 1 versus 2 on-call intensivists and shifts with and without a resident were made using Student's $t$ test for continuous variables and the $\chi^{2}$ test or Fisher's exact test for categorical variables.

Holter recordings were scanned using a MARS Unity Workstation (version 4.0a, GE Marquette Medical Systems) by 1 of 2 senior technologists and by an electrophysiologist. Time- and frequency-domain measures of heart rate variability were compared with published normal values. ${ }^{7}$

The study was reviewed and approved by the hospital research ethics board, and written informed consent was obtained from all of the subjects before their participation.

\section{Results}

Full assessments were conducted during 35 shifts involving 11 consenting senior fellows over the 6-month period ending April 2002. Two of the senior fellows completed their training at the Hospital for Sick Children in December 2001 and were replaced by 3 new senior fellows in January 2002.

During the 6-month study period, 837 children were admitted to the PCCU; $71 \%$ received mechanical ventilation and $4.3 \%$ died. The mean patient census was 20 , and the mean number of children admitted per day was 5.1.

\section{Rostered hours and the PAIRO-OCOTH guidelines}

Two 4-week roster blocks were reviewed; they comprised twelve 4-week blocks during which 1 of 9 senior fellows was on duty. The participants were rostered a mean of

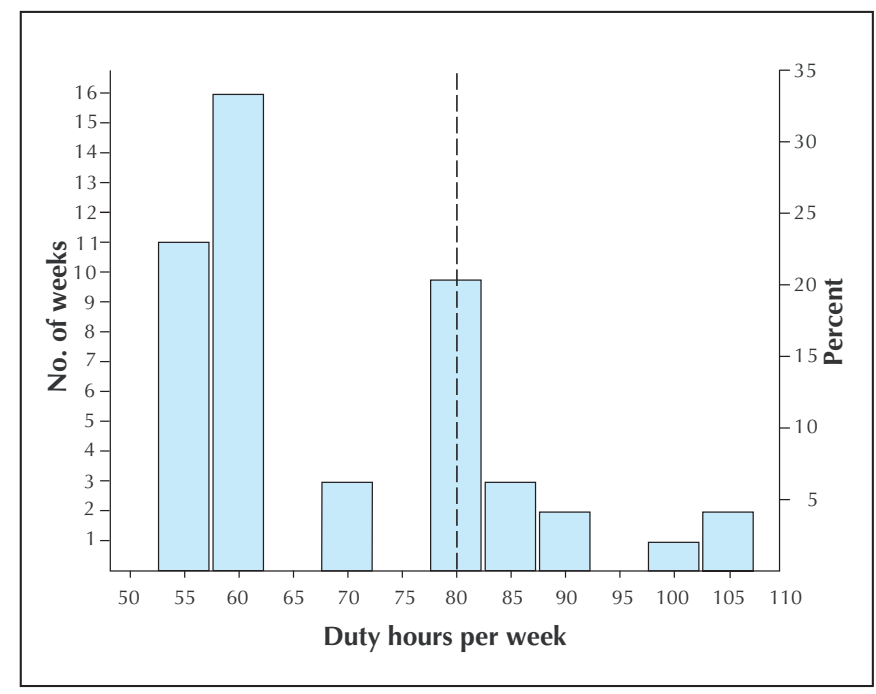

Fig. 1: Duty hours per week of 9 senior fellows working in a tertiary pediatric critical care unit. Two 4-week blocks, totalling 48 rostered weeks, are represented. The bars reflect the number of hours worked per week for the number of weeks noted. For example, the first bar demonstrates that the fellows worked 55 hours per week for 11 of the 48 weeks; the last bar shows that 105 hours were worked per week for 2 of the 48 weeks. 
69 hours (range 55-106) per week. Eight of the 48 weeks involved more than 80 hours rostered for patient care activities (Fig. 1).

The participants were on-call 5-7 days in 28 days, including a maximum of 2 weekend days. They did not work consecutive on-call shifts, and they were relieved of clinical, educational and other responsibilities until the beginning of the next working day.

\section{Workload}

Thirty-five shifts (892 on-call hours) were assessed. The reported mean shift duration was 25.5 hours (range 24-27). The mean patient census at the start of each studied shift was 22 (range 15-32); 56\% of these patients received ventilation. Three children received extracorporeal membrane oxygenation throughout 2 shifts, and 2 children received high-frequency oscillation during another 2 shifts. None of the children received hemodialysis.

A mean of 5.2 children were admitted each day. The participants supervised or admitted 3.8 children (range $0-8$ ) per shift and discharged 1.7 (range 0-4).

The participants recorded a mean of 41 pages per shift; most $(65 \%)$ of the pages were received between $6 \mathrm{pm}$ and the end of the shift (Table 1). Of the pages, $91.3 \%$ originated within the unit, $6.3 \%$ originated within the hospital, and $2.4 \%$ were from other hospitals. The maximum number of documented pages in 1 shift was 87; the minimum was 11 (Fig. 2).

The participants reported performing or supervising at least 1 invasive procedure (intubation, central line, arterial line or chest tube insertion or institution of extracorporeal therapy) during 24 (69\%) of the 35 on-call shifts. Intubation and extubation were the most common procedures (Table 2).

Placement of arterial access and endotracheal intubation occurred on average every second shift and central venous line insertion every fifth. The participants also managed cardiopulmonary resuscitation every seventh shift and were involved in the care of a patient who died once every twelfth on-call shift.

\section{Physiologic stress}

On average, the participants covered $6.3 \mathrm{~km}$ (range

Table 1: Number of pages to the senior fellows during 35 shifts

\begin{tabular}{lcrr}
\hline & \multicolumn{3}{c}{ Time; no. (and \%) of pages } \\
\cline { 2 - 4 } Origin of page & Before $6 \mathrm{pm}$ & After $6 \mathrm{pm}$ & \multicolumn{1}{c}{ All } \\
\hline In-unit & $436(34)$ & $882(66)$ & 1318 \\
In-hospital & $47(52)$ & $44(48)$ & 91 \\
Other hospital & $17(50)$ & $17(50)$ & 34 \\
\hline All & $500(35)$ & $943(65)$ & 1443 \\
\hline
\end{tabular}

$2-11.3 \mathrm{~km}$ ) and took 10660 steps per shift. They reported a mean of 70 minutes for meals and other non-sleep breaks during the shift, including 31 minutes for non-sleep breaks taken between $6 \mathrm{pm}$ and handover time the next morning (7:30 am). In addition, the fellows reported sleeping during $30(86 \%)$ of the shifts, for a mean of 1.9 hours (range $0-4$ hours) (Fig. 3).

Holter monitoring was performed during 9 on-call periods in 6 of the participants. Two demonstrated significant ectopy (1 atrial, symptomatic; 1 ventricular, asymptomatic). All of the recordings showed a predominantly unimodal distribution of heart rates with minimal or no diurnal variation, consistent with minimal or absent sleep during the monitoring interval. All of the recordings revealed a significant (more than 2 standard deviations [SD]) reduction in power spectral density in the high-frequency $(0.15-$ $0.40 \mathrm{~Hz}$ ) band and a shift of the power spectrum into the low-frequency $(0.04-0.15 \mathrm{~Hz})$ band. The resulting lowfrequency:high-frequency ratio (mean 5.6 [SD 1.6]) was also abnormal (normally 1.5-2.0 for adults). Three of the participants demonstrated a significant reduction in SDANN (SD of the average normal R-R intervals for all 5-minute segments). These observations are all consistent with a shift of the normal sympathovagal balance of the heart rate variability spectrum toward sympathetic dominance.

Urinalysis was performed at the end of 33 shifts. Ketones were detected in the urine of participants in $7(21 \%)$ of the 33 shifts. No glycosuria was reported. The mean urine specific gravity was 1.02 (range 1.01-1.03). The 17 urine samples collected from participants at the start of the day shift were negative for ketones ( $p=0.080$, Fisher's exact test).

\section{Staff support}

The mean nurse:patient ratio at the beginning of the shifts was 0.80 (range $0.625-1.0$ ) and was correlated with the pro-

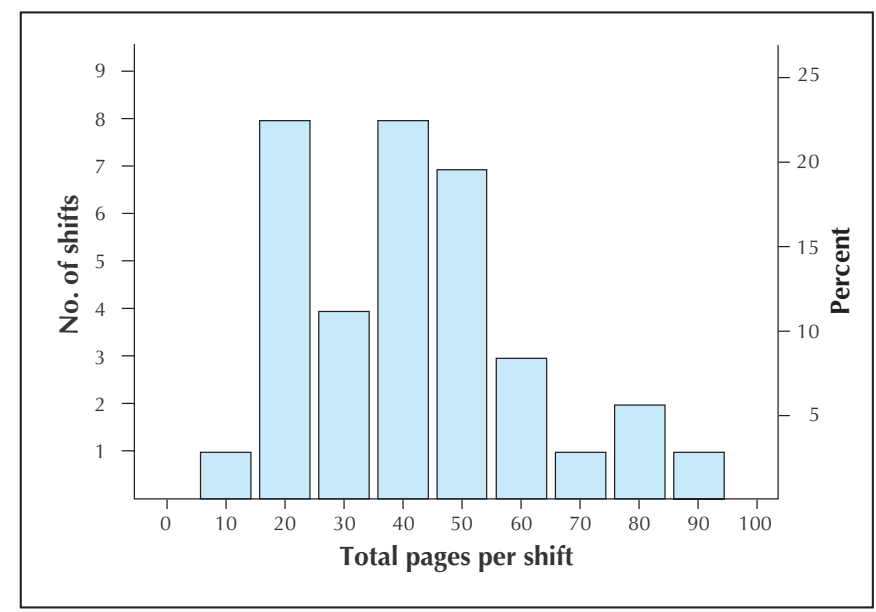

Fig. 2: Number of pages received by the senior fellows during each of 35 on-call, "24-hour" shifts in a tertiary pediatric critical care unit. For example, the second bar demonstrates that, during 8 of the 35 shifts, the fellows received 20 pages per shift. 
portion of patients who were intubated $(r=0.5, p=0.025)$.

Two intensivists were on call for 21 of the 35 shifts, and 1 intensivist was responsible to the unit for the remaining 14 shifts. On average, intensivists departed at 9:06 pm and were away from the unit 9.5 hours per shift (range 3-15 hours). During the time they were away from the unit, they had contact with the senior fellow once every 7.3 hours on average (range 0-4 calls per shift) and returned to the unit after departure in 7 shifts (20\%), for a mean of 1.7 hours a visit (maximum 7 hours).

The presence of a second intensivist was not associated with altered workload or physiologic stress for the senior fellow (as measured by distance walked, stress rating, busyness, urine specific gravity, total calls, sleep hours $[p>0.28$ for all]), but it was associated with more admissions (4.0 v. 5.9 children admitted per shift, $p=0.026$ ).

A second in-house physician, the junior fellow, was present during all of the 35 shifts. In 18 of the shifts, there was also a third in-house physician (a second- or third-year resident on rotation to the unit). The presence of a resident significantly reduced the workload of the participants in terms of distance walked $(5.0 \mathrm{v} .7 .7 \mathrm{~km}, p=0.001)$ and the total mean number of pages during a shift $(34$ v. $50, p=$ 0.012 ), but it did not alter the participants' rating of call stress and busyness, the occurrence of ketonuria or the mean urine specific gravity (Table 3 ).

\section{Interpretation}

This study quantifies the workload, physical demands and personnel support experienced by critical care medicine trainees in a major university-affiliated centre. The trainees in our study worked a mean of 69 hours per week. On-call periods were supported by high nurse:patient ratios and 1 or 2 intensivists, but they were physically demanding: distances of up to $11 \mathrm{~km}$ were covered, break times amounted to 70 minutes on average, and the mean sleeping time was less than 2 hours. The senior fellow was the most senior on-site physician for 9.5 hours of the shift on average and performed or supervised at least 1 invasive procedure in $69 \%$ of the shifts.

Ketonuria was observed in $21 \%$ of the shifts. The measured urine specific gravity suggests that dehydration was common. Although the origins of ketonuria are likely to include dehydration, ongoing exertion and starvation, our findings indicate altered metabolism in response to the oncall stresses.

The number of pages reported by the senior fellows in the study was below that expected by us. This may have been because the generally high workload made it difficult

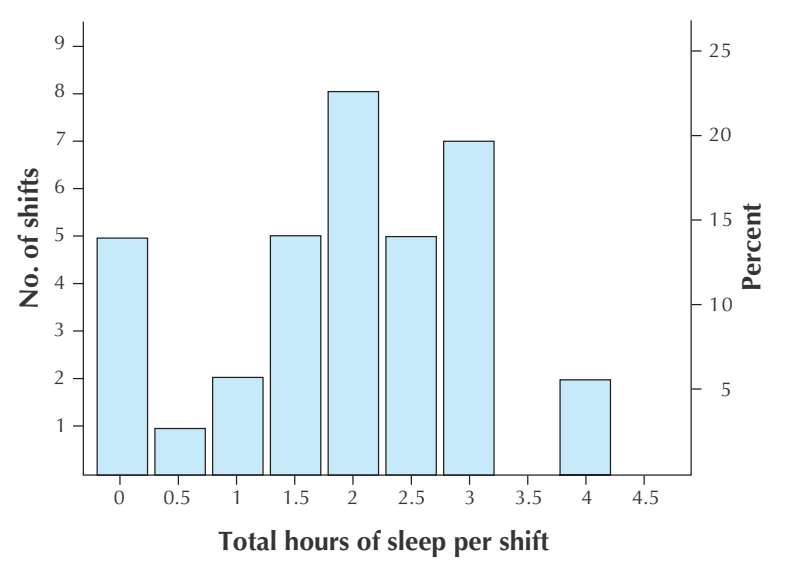

Fig. 3: Total time the senior fellows reported being asleep during each of the 35 shifts. For example, the first bar indicates that the fellows did not sleep during 5 of the 35 shifts; the last bar shows that they slept 4 hours per shift during 2 of the 35 shifts.

Table 2: Number of events and procedures supervised or performed by the senior fellows during 35 on-call shifts

\begin{tabular}{|c|c|c|c|c|}
\hline Procedure or event & $\begin{array}{c}\text { No. } \\
\text { performed }\end{array}$ & $\begin{array}{c}\text { Mean no. } \\
\text { per shift }\end{array}$ & $\begin{array}{l}\text { No. of shifts during } \\
\text { which procedure } \\
\text { performed or event } \\
\text { occurred }\end{array}$ & $\begin{array}{l}\text { Maximum } \\
\text { no. per shift }\end{array}$ \\
\hline Intubation & 16 & 0.46 & 13 & 3 \\
\hline Extubation & 14 & 0.40 & 9 & 3 \\
\hline Intra-arterial catheter insertion & 17 & 0.49 & 16 & 2 \\
\hline Central venous catheter insertion & 7 & 0.20 & 6 & 2 \\
\hline Start ECMO & 1 & 0.03 & 1 & 1 \\
\hline Sternal opening & 3 & 0.09 & 3 & 1 \\
\hline Arrest in PCCU & 5 & 0.14 & 5 & 1 \\
\hline Chest tube insertion & 1 & 0.03 & 1 & 1 \\
\hline Death & 3 & 0.09 & 3 & 1 \\
\hline Start HFO & 3 & 0.09 & 2 & 2 \\
\hline Transports & 5 & 0.14 & 4 & 2 \\
\hline
\end{tabular}

Note: $\mathrm{ECMO}=$ extracorporeal membrane oxygenation, $\mathrm{PCCU}=$ pediatric critical care unit, $\mathrm{HFO}=$ high-frequency oscillation. 
for the fellows to document all of the pages. The nature of the pages was not recorded but will have included "Dr now" overhead calls, less urgent calls to review patients or laboratory results (the majority), administrative calls and nonwork-related calls (rare).

The number of procedures performed or supervised by the participants is probably a conservative estimate. For example, following cardiopulmonary bypass, children are extubated in the unit (2-3 children a day). Extubation may have occurred under the supervision of the on-call intensivist and, thus, was not reported, although the senior fellow would be expected to be involved. Other procedures may have been performed with minimal assistance by competent junior fellows, or less-urgent procedures may have been deferred to the "rested" day staff.

The presence of a rotating resident (as the third in-house physician) was associated with a significant reduction in workload for the senior fellows. The reduced distance walked and number of calls probably reflects the greater division of tasks possible with more personnel and, specifically, the management of "routine" patient care by the resident.

Our study had limitations. With the use of self-reporting, the periods of greatest workload may not have been as well documented as the other periods. Despite this limitation, we believe that the study provides reliable but conservative estimates of workload. The inclusion of formal neuropsychologic testing and measures of error production may also have been of interest; however, the intent of our study was to describe workload and physiologic stress rather than to duplicate previous work demonstrating the linkage between fatigue and reduced performance. ,, $8-11^{-1}$

The fellows we studied were working within the PAIRO-OCOTH guidelines ${ }^{4}$ and the revised American College of Graduate Medical Education (ACGME) ${ }^{5}$ resident duty-hour regulations. However, they did not rest sufficiently to prevent acute sleep deprivation nor, by inference, function optimally throughout the shift. ${ }^{2,8}$ The fellows also exhibited measurable abnormalities in meta-

Table 3: The impact of the presence of a resident on senior fellow's workload

\begin{tabular}{lccc}
\hline & \multicolumn{2}{c}{ Resident present* } & \\
\cline { 2 - 3 } Workload factor & Yes & No & \\
\cline { 2 - 3 } Total calls & 34 & 50 & 0.012 \\
Night-time calls & 24.6 & 30 & 0.244 \\
In-hospital calls & 2.8 & 2.6 & 0.827 \\
Distance walked, km & 5.0 & 7.7 & 0.001 \\
Time asleep, $\mathrm{h}$ & 1.7 & 2.0 & 0.384 \\
Breaks, min & 77 & 68 & 0.524 \\
Self-rating of call stress & 4.1 & 4.3 & 0.652 \\
Self-rating of busyness & 4.6 & 5.0 & 0.424 \\
Ketonuria, no. of shifts & 4 & 3 & 1.00 \\
Specific gravity of urine & 1.02 & 1.02 & 0.997 \\
\hline
\end{tabular}

*All values are means except those for ketonuria, which are the number of shifts in which ketones were detected in the urine of participants. bolism and cardiac rhythm and variability in heart rate.

The consequences are threefold. First, fatigue adversely affects performance, increases propensity for errors and, thus, may cause patient harm. ${ }^{2,910,12}$ Second, trainees' physical and mental health and interpersonal relations may be adversely affected. ${ }^{13-16}$ Finally, training may be compromised. ${ }^{17}$

The current rostering schedule permits trainees to attend most formal education sessions. The clinical impact of these sessions and recollection of their content was not assessed, and the ideal balance has not been well defined. ${ }^{17}$ By most standards, the training program of the Department of Critical Care Medicine at the Hospital for Sick Children is successful. The program is accredited by the Royal College of Physicians and Surgeons of Canada and, over the last 20 years, has trained 176 fellows from 26 countries, including the majority of Canadian intensivists and many pediatric anesthetists.

\section{Summary}

Although within current PAIRO-OCOTH and ACGME regulations, the workload of senior fellows in the Hospital for Sick Children's PCCU is physically demanding, allows limited rest and is associated with sleep deprivation and objective markers of physiologic stress. Judged by the number and destinations of its trainees, this is a highly successful program; however, trainees are unlikely to be performing optimally. This style of health care delivery is almost certainly associated with the production of error and adverse patient outcomes.

Current guidelines and work practices do not mitigate significant fatigue and physical stress. Guideline revision and evaluation of other means to reduce the physical demands on trainees are required if the health care system wishes to address these challenging issues.

This article has been peer reviewed.

From the Department of Critical Care Medicine, Hospital for Sick Children (Parshuram, Dhanani, Kirsh, Cox); and the Division of Cardiology (Kirsh), the Division of Clinical Pharmacology and Toxicology (Parshuram), and the Departments of Paediatrics (Parshuram, Dhanani, Kirsh) and Anaesthesia (Cox), University of Toronto, Toronto, Ont.

Competing interests: None declared.

Contributors: Christopher Parshuram and Joel Kirsh were responsible for conception and design, data acquisition and analysis, and manuscript preparation. Sonny Dhanani was responsible for conception and design, data acquisition and manuscript preparation. Peter Cox was responsible for conception and design and manuscript preparation. All authors approved of the final version.

Acknowledgements: The authors thank Laura Fenwick and Andrea Neilson for their expert processing of the Holter recordings, the fellows who consented to be in the study and the administrative staff of the critical care unit for their assistance with the data.

Christopher Parshuram is a recipient of funding from the Research Institute of the Hospital for Sick Children, Toronto, Ont.

\section{References}

1. Weinger $M B$, Ancoli-Israel S. Sleep deprivation and clinical performance. 7AMA 2002;287(8):955-7.

2. Gaba DM, Howard SK. Patient safety: fatigue among clinicians and the safety 
of patients. N Engl F Med 2002;347(16):1249-55

3. Jha AK, Duncan BW, Bates DW. Fatigue, sleepiness, and medical errors. In: Markowitz AJ, Shojania KG, Duncan BW, McDonald KM, Wachter RM, editors. Making health care safer: a critical analysis of patient safety practices [Evidence Report/Technology Assessment no 43]. San Francisco: Agency for Healthcare Research and Quality; 2003. AHRQ publ. no. 01-E058. Available: www.ahcpr.gov/clinic/ptsafety/ (accessed 2003 Nov 12)

4. PAIRO-OCOTH agreement. 2000 PAIRO guidelines for trainee practice. Toronto: Professional Association of Internes and Residents of Ontario; 2000.

5. Philibert I, Friedmann P, Williams WT, ACGME Work Group on Resident Duty Hours, Accreditation Council for Graduate Medical Education. New requirements for resident duty hours. 7AMA 2002;288(9):1112-4.

6. Veasey S, Rosen R, Barzansky B, Rosen I, Owens J. Sleep loss and fatigue in residency training: a reappraisal. fAMA 2002;288(9):1116-24

7. Task Force of the European Society of Cardiology and the North American Society of Pacing and Electrophysiology. Heart rate variability: standards of measurement, physiological interpretation and clinical use. Circulation 1996;93(5):1043-65.

8. Dawson D, Reid K. Fatigue, alcohol and performance impairment. Nature 1997;388(6639):235.

9. Aya AG, Mangin R, Robert C, Ferrer JM, Eledjam JJ. Increased risk of unintentional dural puncture in night-time obstetric epidural anesthesia. Can $\mathcal{F}$ Anaesth 1999;46(7):665-9.

10. Barth B, Hendey GW, Soliz T. Errors in post-call medication orders. Acad Emerg Med 2001;8(5):468-9.

11. Taffinder NJ, McManus IC, Gul Y, Russell RC, Darzi A. Effect of sleep deprivation on surgeons' dexterity on laparoscopy simulator. Lancet 1998;352 (9135):1191

12. Wu AW, Folkman S, McPhee SJ, Lo B. Do house officers learn from their mistakes? 7AMA 1991;265(16):2089-94.

13. Asken MJ, Raham DC. Resident performance and sleep deprivation: a review. 7 Med Educ 1983;58(5):382-8.

14. Samkoff JS, Jacques CH. A review of studies concerning effects of sleep deprivation and fatigue on residents' performance. Acad Med 1991;66(11):687-93.

15. Steele MT, Ma OJ, Watson WA, Thomas HA Jr, Muelleman RL. The occupational risk of motor vehicle collisions for emergency medicine residents. Acad Emerg Med 1999;6(10):1050-3.

16. Baldwin PJ, Dodd M, Wrate RW. Young doctors' health - I. How do working conditions affect attitudes, health and performance? Soc Sci Med 1997;45(1):35-40.

17. Holzman IR, Barnett SH: The Bell Commission: ethical implications for the training of physicians. Mt Sinai 7 Med 2000;67(2):136-9.

Correspondence to: Dr. Christopher S. Parshuram, Department of Critical Care Medicine, Hospital for Sick Children, Toronto ON M5G 1X8; fax 416 813-7299; chris@sickkids.ca

\section{CMAJ.JAMCél}

CMAJ is powered by HighWire Press - the world leader in online journal publishing.

Search all of MEDLINE and access more than 340 highly cited journals, including CMAJ, the New England

Journal of Medicine, JAMA and BMJ.

\section{Visit WWW.cmaj.ca to sign up for:}

- email table of contents service

- customized alerts on the topics of particular interest to you

- citation alerts on specific articles

\section{WwW.cmaj.ca}

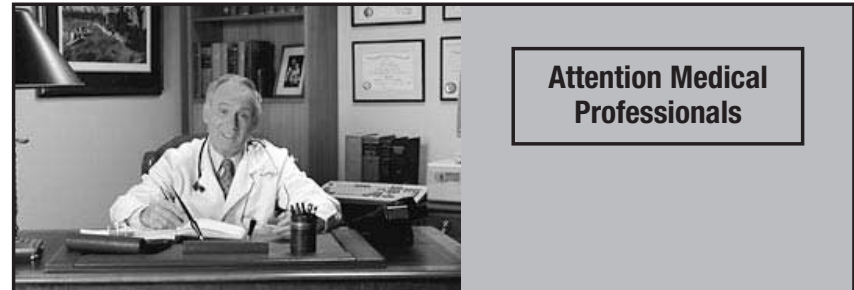

\section{Seminar for Physicians with Tenant Leases Expiring Within 24 Months By Canada's Leading Expert}

Benefits You Will Gain by Attending This Seminar

- Learn how to work with other businesses to reduce your costs

- The ability to recognize and properly deal with risks in your tenant lease

- Alignment of key tenant lease provisions with your retirement plan

- Proper management of the "tenant lease renewal process"

- Elimination of personal guarantees

- Determine what you should be asking for at lease renewal time

The leading provider of education on lease strategies and solutions to physicians across Canada, Cirrus Tenant Lease Services has raised physician awareness of the risks involved with their tenant lease for more than a decade. With overhead costs growing faster than revenues, physicians must look to new and beneficial options for their practices. This workshop will deliver the solutions available to reduce your costs and lower your risks.

All attendants will receive a $F R E E$ lease analysis outlining the hidden pitfalls and risks currently in your lease.

View a video clip of our seminar, visit wWw.cirrustls.com

\section{What Your Colleagues Have Said:}

"A must for every health care professional. Why they did not teach this in school...?" - Dr. Marlon B. Jackson

"This seminar changed my mindset on what is really important in lease negotiations." - Dr. Eric Solberg

"Very eye opening" - Dr. G.S. Lee

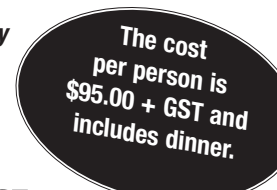

\section{Cirrus}

\section{Upcoming seminars in your area:}

\section{TORONTO}

Wednesday, March 31, 2004

6:30 - 9:30 p.m.

Radisson Toronto Airport

640 Dixon Road, Etobicoke, ON

Thursday, April 1, 2004

6:30 - 9:30 p.m.

Radisson Toronto East

55 Hallcrown PI., North York, ON

\section{VANCOUVER}

Wednesday, May 5, 2004

6:30 - 9:30 p.m

Sheraton Hotel Guilford

15269 104th Avenue, Surrey, BC

Thursday, May 6, 2004

6:30 - 9:30 p.m.

Ramada Inn Vancouver Centre 898 W. Broadway, Vancouver, BC
To register, visit www.cirrustls.com, or call (866) 590-6854 